\title{
CONDITIONS FOR COMMUTATIVE SEMIGROUPS TO HAVE NONTRIVIAL HOMOMORPHISMS INTO NONNEGATIVE (POSITIVE) REALS
}

\author{
YUJI KOBAYASHI
}

\begin{abstract}
Let $S$ be a commutative cancellative semigroup of finite rank (the free rank of the quotient group of $S$ is finite). We give a necessary and sufficient condition for $S$ to have a nontrivial homomorphism into the additive semigroup of nonnegative (positive) reals. We also give a counterexample in the case of infinite rank.
\end{abstract}

1. Introduction and statement of Theorem. Throughout this note "semigroup" means a commutative cancellative semigroup and its operation is written additively. $\mathbf{R}_{0}$ (resp. $\mathbf{R}_{+}$) denotes the additive semigroup of nonnegative (resp. positive) reals. It is a fundamental fact that any $N$ semigroup is homomorphic into $\mathbf{R}_{+}$[4], [5]. What is a necessary and sufficient condition under which a semigroup has a nontrivial homomorphism into $\mathbf{R}_{\mathbf{0}}$ (resp. $\mathbf{R}_{+}$)? This problem is treated in [3] in some special cases. In this note we solve the problem under a certain finiteness condition.

Let $S$ be a semigroup and let $\tilde{S}$ be its quotient group. The rank of $S$ means the free rank of $\tilde{S}$ and is denoted by rank $S . Z_{+}$denotes the set of all positive integers. The theorem we are going to prove is

THEOREM. Let $S$ be a commutative cancellative semigroup of finite rank. Then:

(1) $\operatorname{Hom}\left(S, \mathbf{R}_{0}\right) \neq 0$ if and only if $S$ is not a group.

(2) $\operatorname{Hom}\left(S, \mathbf{R}_{+}\right) \neq \varnothing$ if and only if $S$ satisfies the condition

(*) for any $a, b \in S$ there exists $n \in \mathbf{Z}_{+}$such that lna $\nmid l b^{1}$ for all $l \in \mathbf{Z}_{+}$.

In the last section we give an example; a semigroup $S$ of infinite rank satisfying condition (*) but $\operatorname{Hom}\left(S, \mathbf{R}_{0}\right)=0$ (a fortiori $\left.\operatorname{Hom}\left(S, \mathbf{R}_{+}\right)=\varnothing\right)$.

2. Reduction. Let $\mathbf{Z}$ be the ring of integers and let $\mathbf{Q}$ (resp. $\mathbf{R}$ ) be the rational (resp. real) number field. Assume rank $S=r<\infty$. Let $\tilde{S}$ be the quotient group of $S$ and let $\iota_{1}: S \rightarrow \tilde{S}$ be the injection. By the isomorphism $\tilde{S} \otimes_{\mathbf{z}} \mathbf{Q} \stackrel{\sim}{\rightarrow} \mathbf{Q}^{r}$, we identify $\tilde{S} \otimes_{\mathbf{z}} \mathbf{Q}$ and $\mathbf{Q}^{r}$. Let $\iota_{2}: \tilde{S} \rightarrow \tilde{S} \otimes_{\mathbf{Z}} \mathbf{Q}$ be the

Received by the editors August 12, 1976.

AMS (MOS) subject classifications (1970). Primary 20M15.

Key words and phrases. Commutative cancellative semigroup, quotient group, rank, convex cone.

${ }^{1}$ We write $a \mid b$ if either $a=b$ or $a+c=b$ for some $c \in S$, and otherwise we write $a \nmid b$. 
canonical mapping. Let $\iota=\iota_{1} \circ \iota_{2}$ and $C_{S}=\left\{a \in \mathbf{Q}^{r} \mid\right.$ na $\in \iota(S)$ for some $\left.n \in \mathbf{Z}_{+}\right\}$.

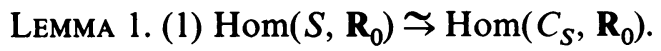

(2) $\operatorname{Hom}\left(S, \mathbf{R}_{+}\right) \stackrel{\sim}{\rightarrow} \operatorname{Hom}\left(C_{S}, \mathbf{R}_{+}\right)$.

Proof. $f \in \operatorname{Hom}\left(S, \mathbf{R}_{0}\right)$ is extended uniquely to $\tilde{f} \in \operatorname{Hom}(\tilde{S}, \mathbf{R})$ in the natural way. $\tilde{f}$ is extended uniquely to $\bar{f} \in \operatorname{Hom}\left(\tilde{S} \otimes_{\mathbf{Z}} \mathbf{Q}, \mathbf{R}\right)$ by $\bar{f}(s \otimes q)=$ $\tilde{f}(s) q$. It is easy to see $\bar{f} \geqslant 0$ on $C_{S}$, and, moreover, $\bar{f}>0$ on $C_{S}$ if $f>0$. This proves the lemma.

LEMMA 2. $S$ is a group if and only if $C_{S}$ is a group.

Proof. If $S$ is a group, $\iota(S)$ is a group. It is easy to prove that $C_{S}$ is a group. Conversely, assume $C_{S}$ is a group. Then for any $s \in S$ there exists $x \in C_{S}$ such that $s \otimes 1+x=0$. By the definition of $C_{s}, n x=s^{\prime} \otimes 1 \in \iota(S)$ for some $n \in \mathbf{Z}_{+}$and some $s^{\prime} \in S$. Hence $\left(n s+s^{\prime}\right) \otimes 1=0$. Therefore there exists $m \in \mathbf{Z}_{+}$such that $m\left(n s+s^{\prime}\right)=0$. This shows $(m n-1) s+m s^{\prime}$ is the inverse element of $s$. Thus every element of $S$ is invertible, and then $S$ is a group.

The proof of the following lemma is routine and we omit it.

LEMMA 3. S satisfies condition (*) in $\S 1$ if and only if $C_{S}$ satisfies the condition

$$
\bigcap_{n=1}^{\infty}\left(n x+C_{s}\right)=\varnothing \quad \text { for all } x \in C_{s} \text {. }
$$

By the definition of $C_{S}, C_{S}$ is a subsemigroup of $\mathbf{Q}^{r}$ satisfying that $n x \in C_{S}$ for some $n \in \mathbf{Z}_{+}$implies $x \in C_{S}$. That is, $C_{S}$ is a convex cone in $\mathbf{Q}^{r}$.

3. Convex cones in $Q^{r}$ and $\mathbf{R}^{r}$. Let $S$ be a convex cone in $\mathbf{Q}^{r}$. $\mathbf{Q}^{r}$ is considered as a subset of $\mathbf{R}^{r}$ naturally. Let $\bar{S}$ be the closure of $S$ in $\mathbf{R}^{r}$ (we always consider the usual topology of $\mathbf{R}^{r}$ ). Then $\bar{S}$ is a closed convex cone in $\mathbf{R}^{r}$.

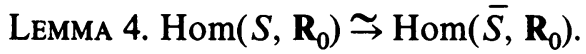

Proof. $f \in \operatorname{Hom}\left(S, \mathbf{R}_{\mathbf{0}}\right)$ can be extended to $\tilde{f} \in \operatorname{Hom}(\tilde{S}, \mathbf{R})$, and $\tilde{f}$ can be extended to $F \in \operatorname{Hom}\left(\mathbf{Q}^{r}, \mathbf{R}\right)$ since $\mathbf{R}$ is a divisible abelian group (see [2] for example). From the isomorphism $\operatorname{Hom}\left(\mathbf{Q}^{r}, \mathbf{R}\right) \stackrel{\sim}{\rightarrow} \mathbf{R}^{r}$, it follows that $F$ (consequently $f$ ) is continuous. Therefore $f$ is extended uniquely to $\bar{f} \in$ $\operatorname{Hom}\left(\bar{S}, \mathbf{R}_{0}\right)$.

Lemma 5. Let $A$ be a convex set of $\mathbf{Q}^{r}$ and let $A^{\prime}$ be the convex hull of $A$ in $\mathbf{R}^{r}$. Then $A^{\prime} \cap \mathbf{Q}^{r}=A$.

LEMMA 6. $S$ is a group if and only if $\bar{S}$ is a group.

Proof. It is easy to see that $\bar{S}$ is a group if $S$ is a group. Conversely, assume that $\bar{S}$ is a group. Since $\bar{S}$ is an $\mathbf{R}$-vector subspace of $\mathbf{R}^{r}$, we may assume 
$\bar{S}=\mathbf{R}^{r}$. Since $S$ is dense in $\mathbf{R}^{r}$, it follows that $S^{\prime}=\mathbf{R}^{r}$. By Lemma 5 , $S=S^{\prime} \cap \mathbf{Q}^{r}=\mathbf{Q}^{r}$, thus $S$ is a group.

$I(\bar{S})$ denotes the subgroup of $\bar{S}$ of all invertible elements of $\bar{S}$. Obviously $I(\bar{S})$ is an $\mathbf{R}$-vector subspace of $\mathbf{R}^{r}$.

Lemma 7. $I(\bar{S}) \cap S=\left\{a \in S \mid \cap_{n=1}^{\infty}(n a+S) \neq \varnothing\right\}$.

Proof. Assume that $b \in \cap_{n=1}^{\infty}(n a+S) \neq \varnothing$. Then $b-n a \in S$ for all $n \in \mathbf{Z}_{+}$. Let $c_{n}=b / n-a$. Then $c_{n} \in S$. Since $c_{n} \rightarrow-a$ as $n \rightarrow \infty$, it follows that $-a \in \bar{S}$. This shows $a \in I(\bar{S}) \cap S$. Conversely let $a \in I(\bar{S}) \cap$ $S$. Let $L$ be the hyperplane through $-a$ perpendicular to the line through $a$ and the origin. $L \cap S$ is convex and dense in $L \cap \bar{S}$. By Lemma 8, which we state below, there exists $b \in L \cap S$ such that $(b-n a) /(n+1) \in L \cap S$ for all $n \in \mathbf{Z}_{+}$. Hence $b-n a \in S$ for all $n \in \mathbf{Z}_{+}$, that is, $b \in \bigcap_{n=1}^{\infty}(n a+S) \neq$ $\varnothing$. This completes the proof.

Lemma 8. Let $A$ be a convex set of $\mathbf{Q}^{r}$ and let $\bar{A}$ be the closure of $A$ in $\mathbf{R}^{r}$. Let $a \in \bar{A} \cap \mathbf{Q}$. Then there exists $b \in A$ such that every rational point of the open segment joining $a$ and $b$ is contained in $A$.

Proof. Let $A^{\prime}$ be the convex hull of $A$ in $\mathbf{R}^{r}$. If $A^{\prime}$ has an inner point, we can choose an element $b$ of $A$ which is an inner point of $A^{\prime}$ since $A$ is dense in $A^{\prime}$. Then it is known that the open segment joining $a$ and $b$ is contained in $A^{\prime}$ (see [1, Proposition 16, p. 54]). Hence every rational point of the segment is contained in $A^{\prime} \cap \mathbf{Q}^{r}=A$. If $A^{\prime}$ has no inner point, then $A$ does not contain $r+1$ points in general position. Therefore $A$ is contained in a hyperplane of $\mathbf{Q}^{r}$. Thus the proof is reduced to the case of dimension $r-1$. Repeating this, we may only prove the lemma in the case of $r=1$, but in this case the assertion of the lemma is clear. The proof is completed.

4. Proof of Theorem. The necessity of the conditions of the Theorem is clear. By virtue of Lemmata 1, 2, 4 and 6, in order to prove (1) of the Theorem we may show for a convex cone $S$ in $\mathbf{Q}^{r}$ that

$\left(1^{\prime}\right)$ if $\bar{S}$ is not a group, then $\operatorname{Hom}\left(\bar{S}, \mathbf{R}_{0}\right) \neq 0$.

By virtue of Lemmata 1, 3 and 7, in order to prove (2) of the Theorem we may show for a convex cone $S$ in $\mathbf{Q}^{r}$ that

$\left(2^{\prime}\right)$ if $I(\bar{S}) \cap S=\varnothing$, then $\operatorname{Hom}\left(S, \mathbf{R}_{+}\right) \neq \varnothing$.

Let $\eta: \mathbf{R}^{r} \rightarrow \mathbf{R}^{r} / I(\bar{S})$ be the canonical surjection to the quotient space. Then $\eta(\bar{S})$ is a closed convex cone in $\mathbf{R}^{r} / I(\bar{S})$ satisfying $I(\eta(\bar{S}))=0$. Then $\eta(\bar{S})$ is convex strictly at the origin, hence contained strictly in a half-space of $\mathbf{R}^{r} / I(\bar{S})$ except the origin. Therefore, there exists a functional $\varphi$ of $\mathbf{R}^{r} / I(\bar{S})$ such that $\varphi(x)>0$ for all $x \in \eta(\bar{S}) \backslash\{0\}$. Let $f=(\varphi \circ \eta) \mid \bar{s}$, then $f \in$ $\operatorname{Hom}\left(\bar{S}, \mathbf{R}_{0}\right)$ and $f(a)>0$ for all $a \in \bar{S} \backslash I(\bar{S})$. If $\bar{S}$ is not a group, then $\bar{S} \backslash I(\bar{S}) \neq \varnothing$, hence $f \neq 0$. Therefore $\operatorname{Hom}\left(\bar{S}, \mathbf{R}_{0}\right) \neq 0$, this proves $\left(1^{\prime}\right)$. If $\bar{S}$ satisfies $I(\bar{S}) \cap S=\varnothing$, then $\left.f\right|_{S}>0$. Therefore $\operatorname{Hom}\left(S, \mathbf{R}_{+}\right) \neq \varnothing$; this proves $\left(2^{\prime}\right)$. The proof of the Theorem is complete. 
5. Counterexample in the case of infinite rank. In the case of infinite rank, condition (*) in the Theorem does not imply $\operatorname{Hom}\left(S, \mathbf{R}_{0}\right) \neq 0$ as the following example $S$ shows. $S$ is constructed as a subsemigroup of the free group $\bigoplus_{1}^{\infty} \mathbf{Z}$ of countable rank. Let $T_{n}$ be an $n \times n$-matrix:

$$
T_{n}=\left(\begin{array}{cccc}
\left(\begin{array}{c}
n-1 \\
n-1
\end{array}\right) & \left(\begin{array}{c}
n-1 \\
n-2
\end{array}\right) & \cdots & \left(\begin{array}{c}
n-1 \\
0
\end{array}\right) \\
& \left(\begin{array}{c}
n-2 \\
n-2
\end{array}\right) & \cdots & \left(\begin{array}{c}
n-2 \\
0
\end{array}\right) \\
& & \ddots & \vdots \\
& 0 & & \left(\begin{array}{c}
0 \\
0
\end{array}\right)
\end{array}\right),
$$

where (.) denotes the binomial coefficients.

For $x={ }^{t}\left(x_{1}, x_{2}, \ldots, x_{i}, \ldots\right) \in \bigoplus_{1}^{\infty} Z$ or for $x={ }^{t}\left(x_{1}, x_{2}, \ldots, x_{m}\right) \in$ $\bigoplus_{1}^{m} Z$ with $m>n$, we define $\tau_{n}(x)={ }^{t}\left(x_{1}, x_{2}, \ldots, x_{n}\right)$ and $\pi_{n}(x)=x_{n}$. Set $S(n)=\left\{x \in \bigoplus_{1}^{\infty} Z \mid T_{n} \tau_{n}(x)>0\right.$, and $\pi_{m}(x)=0$ for $\left.m>n\right\}$, where $T_{n} \tau_{n}(x)$ $>0$ means that all components of $T_{n} \tau_{n}(x)$ are positive. Now we define $S=\cup_{n=1}^{\infty} S(n) \subset \bigoplus_{1}^{\infty} Z$.

$S$ is a semigroup: We define $\bar{S}(n)=\left\{x \in \bigoplus_{1}^{\infty} Z \mid T_{n} \tau_{n}(x)>0\right.$, and $\pi_{m}(x)=$ 0 for $m>n$ \}. By a simple calculation using the formula

$$
\left(\begin{array}{l}
n \\
r
\end{array}\right)=\left(\begin{array}{l}
n-1 \\
r-1
\end{array}\right)+\left(\begin{array}{l}
n-2 \\
r-1
\end{array}\right)+\cdots+\left(\begin{array}{l}
r-1 \\
r-1
\end{array}\right)
$$

we find $\bar{S}(n) \subset \bar{S}(m)$ if $m>n$. Hence $S(m)+S(n) \subset S(m)+\bar{S}(m) \subset$ $S(m)$. This shows that $S$ is closed with respect to addition, so $S$ is a semigroup.

$S$ satisfies condition (*): Let $a \in S(p)$ and $b \in S(q)$. If $p>q$, then clearly $l(b-a) \notin S$ for all $l \in \mathbf{Z}_{+}$. If $p \leqslant q$, then $\pi_{p}(a)>0$, and $\pi_{p^{\prime}}(a)=0$ for $p^{\prime}>p$. Hence $\pi_{p}\left(T_{q} \tau_{q}(a)\right)=\pi_{p}(a)>0$. If we choose $n \in \mathbf{Z}_{+}$such that $n>\pi_{p}\left(T_{q} \tau_{q}(b)\right) / \pi_{p}(a)$, then

$$
\pi_{p}\left(T_{q} \tau_{q}(l(b-n a))\right)=l\left(\pi_{p}\left(T_{q} \tau_{q}(b)\right)-n \pi_{p}(a)\right)<0
$$

for all $l \in \mathbf{Z}_{+}$. This implies $l(b-n a) \notin S$ for all $l \in \mathbf{Z}_{+}$. Thus we see in either case that $S$ satisfies condition (*).

$\operatorname{Hom}\left(S, \mathbf{R}_{0}\right)=0$ : We define $\bar{S}=\cup_{n=1}^{\infty} \bar{S}(n)$. Since $f \in \operatorname{Hom}\left(S(n), \mathbf{R}_{0}\right)$ is extended uniquely to $\bar{f} \in \operatorname{Hom}\left(\bar{S}(n), \mathbf{R}_{0}\right), \varphi \in \operatorname{Hom}\left(S, \mathbf{R}_{0}\right)$ is extended uniquely to $\bar{\varphi} \in \operatorname{Hom}\left(\bar{S}, \mathbf{R}_{0}\right)$. Hence it suffices to prove $\operatorname{Hom}\left(\bar{S}, \mathbf{R}_{0}\right)=0$. Let $n_{0} \in \mathbf{Z}_{+}$and let $x \in \bigoplus_{1}^{\infty} \mathbf{Z}$ satisfy that $\pi_{n_{0}}(x)>0$ and $\pi_{n}(x)=0$ for $n>n_{0}$. Then we easily have $T_{m} \tau_{m}(x) \geqslant 0$ for a sufficiently large $m$, hence $x \in \bar{S}(m) \subset \bar{S}$. Therefore, for any $a \in \bar{S}$ (say $a \in \bar{S}(p)$ ) there exists $b \in S$ (any $b \in S(p+1)$ is available) such that $l a \mid b$ in $\bar{S}$ for all $l \in \mathbf{Z}_{+}$. From this it follows immediately that $\operatorname{Hom}\left(\bar{S}, \mathbf{R}_{0}\right)=0$.

\section{REFERENCES}

1. N. Bourbaki, Éléments de mathématique. Fasc. XV. Livre V: Espaces vectoriels topologiques, Actualités Sci. Indust. No. 1189, Hermann, Paris, 1966. MR 34 \#3277. 
2. L. Fuchs, Infinite abelian groups. Vol. I, Academic Press, New York, 1970. MR 41 \#333.

3. H. B. Hamilton, T. E. Nordahl and T. Tamura, Commutatioe cancellatioe semigroups without idempotents, Pacific J. Math. 61 (1975), 441-456.

4. Y. Kobayashi, Homomorphisms on $\mathbf{N}$-semigroups into $\mathbf{R}_{+}$and the structure of $\boldsymbol{N}$-semigroups, J. Math. Tokushima Univ. 7 (1973), 1-20. MR 48 \#3831.

5. T. Tamura, Irreducible $\Re$-semigroups, Math. Nachr. 63 (1974), 71-88. MR 51 \#3336.

Department of Mathematics, Faculty of Education, Toxushma University, TOKUSHMA, JAPAN 